\title{
Determinación de las propiedades térmicas de un mortero modificado con sargazo como material alternativo en construcción
}

\section{Determination of the thermal properties of a modified mortar with sargassum as an alternative material in construction}

\author{
ZAVALA-ARCEO, Alberto ${ }^{1} \dagger$, CRUZ-ARGÜELLO, Julio César ${ }^{*}$, FIGUEROA-TORRES, Mayra \\ Zyzlila $^{2}$ y YELADAQUI-TELLO, Alberto ${ }^{1}$
}

${ }^{1}$ Tecnológico Nacional de México/Instituto Tecnológico de Chetumal, Av. Insurgentes 330 C.P. 77013 Col. David Gustavo Gtz. Chetumal, Q.Roo, México

${ }^{2}$ Universidad Autónoma de Nuevo León, Facultad de Ingeniería Civil - IIC, Av. Universidad S/N, Ciudad Universitaria CP 66455 San Nicolás de los Garza, Nuevo León, México

ID $1^{\text {er }}$ Autor: Alberto, Zavala-Arceo / ORC ID: 0000-0001-5504-3807, CVU CONACYT ID: 863313

ID $1^{\text {er }}$ Coautor: Julio César, Cruz-Argüello / ORC ID: 0000-0001-8664-9422, CVU CONACYT ID 221002

ID $2^{\text {do }}$ Coautor: Mayra Zyzlila, Figueroa-Torres / ORC ID: 0000-0002-68237384, CVU CONACYT ID 42436

ID $3^{\text {er }}$ Coautor: Alberto, Yeladaqui-Tello / ORC ID: 0000-0003-3140-9522, CVU CONACYT ID: 200680

DOI: $10.35429 /$ JCE.2019.10.3.1.9

Recibido 03 de Septiembre, 2019; Aceptado 09 Noviembre, 2019

\begin{abstract}
Resumen
En el presente trabajo se analizan las propiedades de un mortero con agregado de alga de sargazo tratada, en sustitución del agregado pétreo en porcentajes de $5 \%$, $10 \%, 15 \%$ y $20 \%$ con el fin de reducir la conductividad térmica del recubrimiento y la carga térmica del interior de las edificaciones, disminuyendo de esta manera el consumo energético e implementando materiales sustentables a la industria de la construcción. Para propósitos de esta investigación se realizó una caracterización de los componentes del mortero, el agregado pétreo y el sargazo tratado para su uso como agregado. Se elaboraron muestras para cada porcentaje y se realizaron pruebas de resistencia a la compresión a los $3,7,14$ y 28 días para analizar el comportamiento del mortero durante el proceso de endurecimiento. Se realizaron pruebas de adherencia para determinar si las mezclas son eficientes para su uso como recubrimiento. Posteriormente se evaluó la transferencia térmica por conducción y convección. El material demostró características hidrofílicas por lo cual, los análisis de las propiedades mecánicas demostraron a mayor sustitución de sargazo, una reducción en la resistencia, limitando los porcentajes eficientes al 5 y $10 \%$ para su uso.
\end{abstract}

Mortero, Aislante térmico, Sargazo

\begin{abstract}
In the present work we analyze the properties of a mortar with the addition of treated sargassum algae, replacing the stone aggregate in percentages of $5 \%, 10 \%, 15 \%$ and $20 \%$ in order to reduce the thermal conductivity of the coating and the thermal load of the interior of the buildings, thus reducing energy consumption and implementing sustainable materials to the construction industry. For the purposes of this investigation, a characterization of the components of the mortar was carried out, the stone aggregate and the sargassum treated for its use as an aggregate. Samples were prepared for each percentage and compression resistance tests were performed at 3, 7, 14 and 28 days to analyze the behavior of the mortar during the hardening process. Adhesion tests were performed to determine if the mixtures are efficient for use as a coating. Subsequently, the thermal transfer was evaluated by conduction and convection. The material showed hydrophilic characteristics, therefore, the analysis of the mechanical properties showed as bigger substitution of sargassum, low is the resistance, limiting the efficient percentages to 5 and $10 \%$ for its use.
\end{abstract}

Mortar, Thermal insulator, Sargassum

Citación: ZAVALA-ARCEO, Alberto, CRUZ-ARGÜELlO, Julio César, FIGUEROA-TORRES, Mayra Zyzlila y YELADAQUI-TELLO, Alberto. Determinación de las propiedades térmicas de un mortero modificado con sargazo como material alternativo en construcción. Revista de Ingeniería Civil. 2019 3-10: 1-9

\footnotetext{
* Correspondencia del Autor (correo electrónico: jcruz@itchetumal.edu.mx)

$\dagger$ Investigador contribuyendo como primer autor.
} 


\section{Introducción}

El uso de aislantes térmicos en la construcción se ha convertido en una necesidad en zonas con climas extremos para asegurar un confort para las personas y una mejor calidad de vida. El aislamiento integrado en los materiales es una de las soluciones implementadas para reducir significativamente la carga de calor en edificación (Osseni, 2017) Por otro lado su estudio y aplicación en la edificación contribuye al uso eficiente de la energía, teniendo una reducción en la energía utilizada en los equipos de ventilación que se utilizan para obtener el confort que las personas necesitan, los cuales representan un porcentaje significativo del consumo energético de las edificaciones.

El estudio y elaboración de los aislantes térmicos ha avanzado desde su aparición y han surgido diferentes tipos de aplicaciones y de materiales, de estos surgen los denominados "bio-aislantes" los cuales cumplen la misma función desde un ámbito más sustentable, el estudio de los bio-aislantes comenzó a partir de la primera publicación de este tema en 1974, sin embargo el desarrollo de este no fue muy desarrollado hasta el año 1998 cuando comenzaron a surgir más investigaciones (Liu, 2017).

De entre estos podemos destacar los morteros térmicos con materiales orgánicos procesados, los cuales por lo general son residuos de actividades o desperdicios. Se han realizado investigaciones de morteros térmicos con diferentes materiales orgánicos tratados, tales como madera (Corinaldesi, 2016), fibras de palma (Benmansour, 2014), cascaras de plátano (Osseni, 2016) entre otras, las cuales sustentan que el uso de un material orgánico debidamente tratado, es viable como material de sustitución en morteros para mejorar sus propiedades térmicas. En la región se utilizan comúnmente morteros como recubrimiento de las viviendas y edificaciones en general, estos tienen un papel fundamental en el flujo de calor hacia el interior de las edificaciones ya que son los primeros en recibir los rayos solares antes de los demás elementos (Puc-Ortiz, 2009). La economía del estado de Quintana Roo se basa en la industria turística que contribuye considerablemente al producto interno bruto del país, las costas del estado han recibido cantidades masivas atípicas de las macroalgas pardas pelágicas Sargassum fluitans y $S$. natans desde el verano de 2014.
La acumulación y descomposición de algas en playas y aguas litorales afecto los ecosistemas costeros, la salud humana y la industria turística, siendo esta ultima el motor económico del estado (Rodríguez, 2017). Esta alga genera una contaminación visual al acumularse en la costa y una contaminación odorífera al descomponerse. (Tussenbroek, 2017).

El alga de sargazo cuenta con propiedades de aislamiento térmico debido a su conductividad térmica de $0.045 \mathrm{~W} / \mathrm{mK}$ y su valor de calor especifico de $2000 \mathrm{~J} / \mathrm{kgK}$ y de acuerdo al análisis prospectivo del alga (Fernández, 2017) es teóricamente adecuado para su uso en construcción. Según Asdrubali (2015) para que un material sea considerado un aislante térmico estos valores deben de permanecer menores a $0.07 \mathrm{~W} / \mathrm{mK}$ y mayores a $1400 \mathrm{~J} / \mathrm{kgK}$ respectivamente lo cual hace de este material teóricamente apto para su uso como aislante térmico. Basándose en las propiedades de esta alga y la disponibilidad que existe en el estado, se propone darle un uso como material de construcción, convirtiendo este residuo en un beneficio social. Para ello se desarrollaran pruebas mecánicas y térmicas para determinar el comportamiento del alga como material de sustitución al agregado pétreo del mortero y comprobar la viabilidad de su uso.

\section{Parte experimental}

\section{Materiales}

El mortero con el que se realizaron las pruebas está compuesto por cemento portland, agua, agregado pétreo de la región y una sustitución de sargazo previamente tratado en porcentajes de 5, 10,15 y $20 \%$; los cuales fueron comparados con un mortero regular para determinar su eficiencia.

\section{Sargazo}

El sargazo fue recolectado en su estado natural en las costas y lavado con agua a una temperatura con el objetivo de eliminar impurezas superficiales, posterior mente se realizó un lavado químico el cual consistió en la inmersión del material en una solución de agua destilada e hidróxido de sodio $(\mathrm{NaOH})$ en proporciones de $40 \mathrm{~g}$ por cada litro de agua (fig. 1), por último se sometió el material a un proceso de deshidratación (fig. 2-A) y se trituró con el fin de obtener una granulometría fina similar a el agregado comúnmente utilizado en la zona y el cual será sustituido parcialmente (fig. 2-B). 

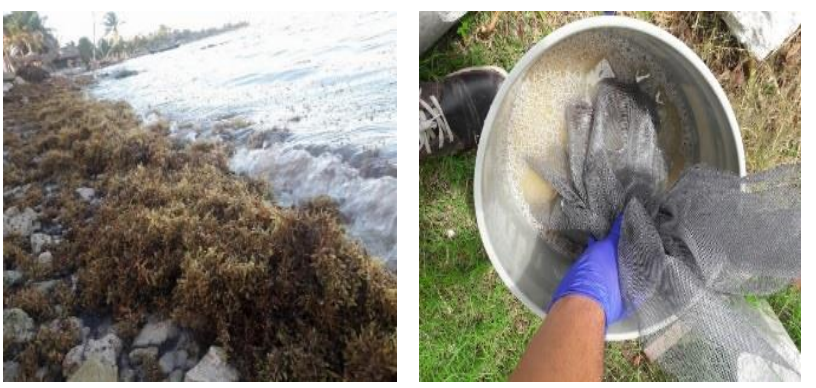

Figura 1 Recolección y lavado del material
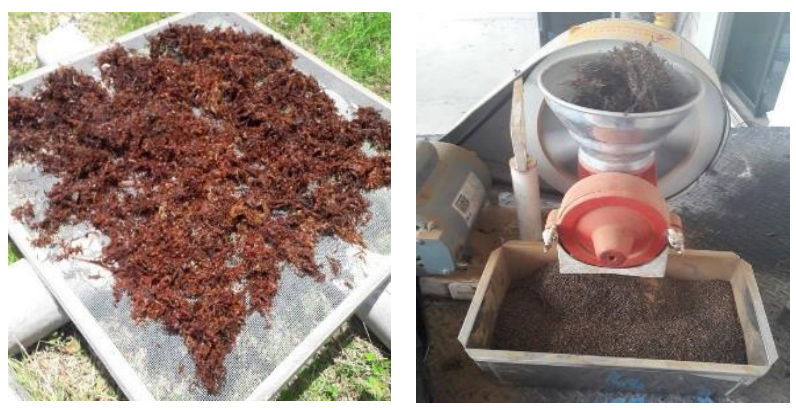

Figura 2 Deshidratación y triturado del material

Se realizó la caracterización del material resultante obteniendo su granulometría, peso volumétrico, peso específico seco suelto, peso específico seco compacto, contenido de humedad y capacidad de absorción. Esta caracterización se llevó a cabo bajo los mismos procedimientos y normativas utilizados en la caracterización del material pétreo.

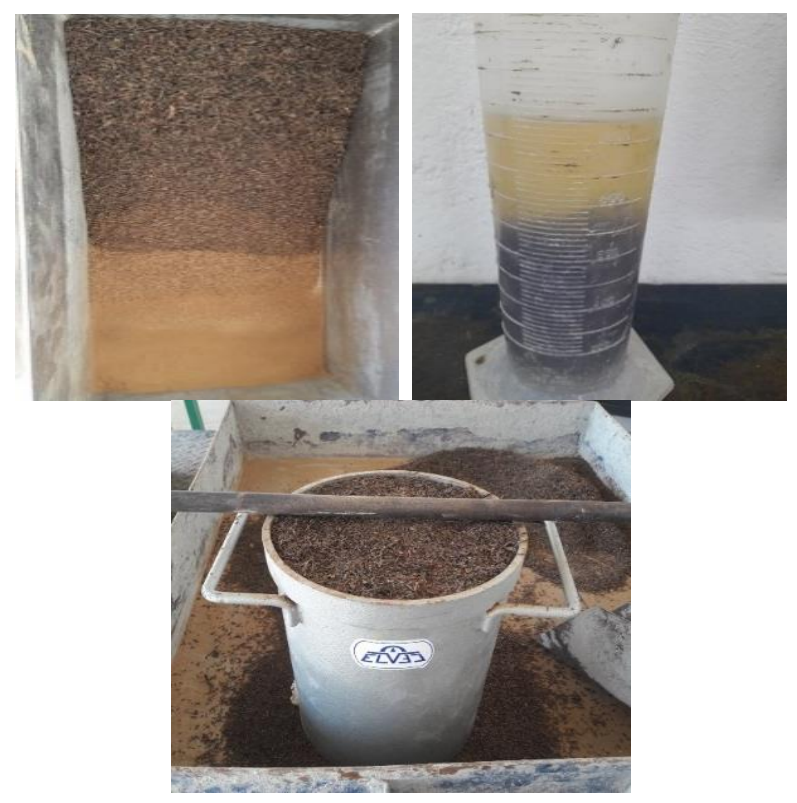

Figura 3 Caracterización del material de sargazo

\section{Agregado Pétreo}

Se realizó la caracterización del material pétreo como agregado en la región, el cual es determinado comúnmente utilizado como piedra caliza extraída de bancos de material y triturada como agregado fino.
De igual manera se realizó la caracterización del material, obteniendo su granulometría, peso volumétrico, peso específico seco suelto, peso específico seco compacto, contenido de humedad y capacidad de absorción.
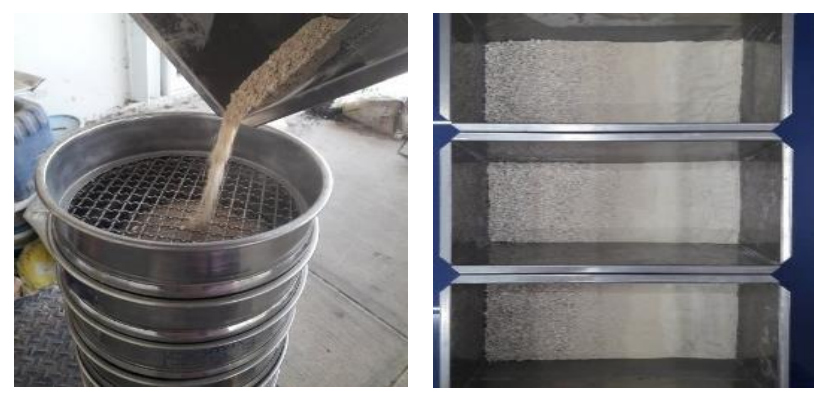

Figura 4 Granulometría del agregado pétreo
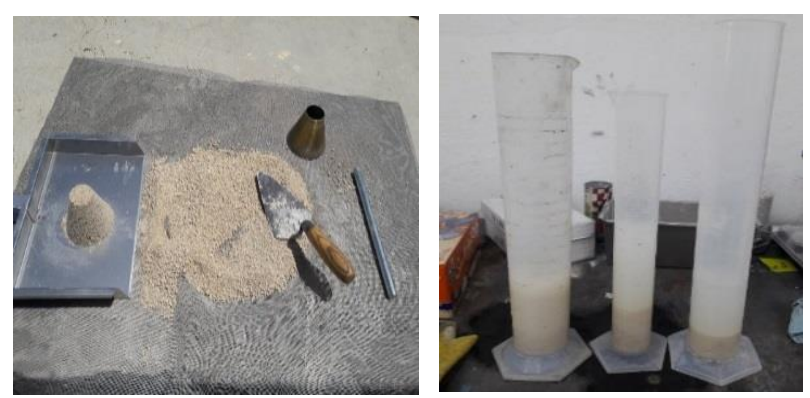

Figura 5 Caracterización del material pétreo

\section{Dosificación}

De acuerdo con el manual de dosificación de morteros, las cantidades de material para un mortero diseñado para ser utilizado como recubrimiento de muros son las que se muestran en la tabla 1. El mortero se diseñó con una resistencia de diseño de $75 \mathrm{~kg} / \mathrm{cm}^{3}$, una fluidez de entre $100 \%$ y $120 \%$ y con una relación agua/cemento de 0.99 .

\begin{tabular}{|l|r|r|r|r|r|}
\hline \multicolumn{7}{|c|}{ Dosificación de material por metro cúbico de } \\
mortero $(\mathbf{K g})$ \\
\hline \multirow{2}{*}{ Material } \\
\cline { 2 - 6 } & $0 \%$ & $5 \%$ & $10 \%$ & $15 \%$ & $20 \%$ \\
\hline Cemento & 325 & 325 & 325 & 325 & 325 \\
\hline Agua & 320.35 & 320.35 & 320.35 & 320.35 & 320.35 \\
\hline $\begin{array}{l}\text { Agregado } \\
\text { fino }\end{array}$ & 1669.93 & 1586.44 & 1502.94 & 1419.44 & 1335.95 \\
\hline Sargazo & 0.000 & 27.909 & 55.818 & 83.726 & 111.635 \\
\hline
\end{tabular}

Tabla 1 Dosificación de los materiales

\section{Elaboración de muestras}

Una vez definidas las proporciones de cada material y en las diferentes dosificaciones, se prosiguió a elaborar las muestras para las pruebas mecánicas y térmicas.

ZAVALA-ARCEO, Alberto, CRUZ-ARGÜELLO, Julio César, FIGUEROA-TORRES, Mayra Zyzlila y YELADAQUI-TELLO, Alberto. Determinación de las propiedades térmicas de un mortero modificado con sargazo como material alternativo en construcción. Revista de Ingeniería Civil. 2019 
Para las pruebas mecánicas se elaboraron cubos de concreto de $5 \mathrm{~cm}$ de espesor.

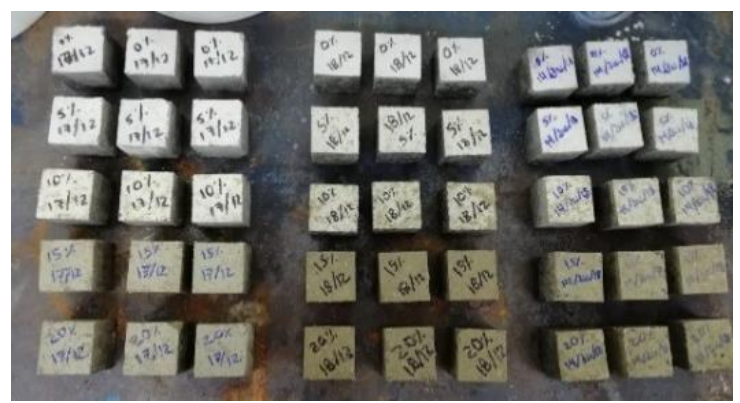

Figura 6 Elaboración de muestras para pruebas mecánicas

Para las pruebas térmicas se elaboraron muestras por cada porcentaje de sustitución, en 2 pruebas de conductividad, una prueba por conducción y otra por convección. Las pruebas de conducción se hicieron con muestras de mortero circulares con 2 " de diámetro y $1 / 2$ “de espesor (Fig. 7- A) y las pruebas de convección se realizaron con muestras rectangulares de $30 \mathrm{~cm}$ de largo, $10 \mathrm{~cm}$ de ancho y $1 / 2$ “de espesor (fig. 7-B).
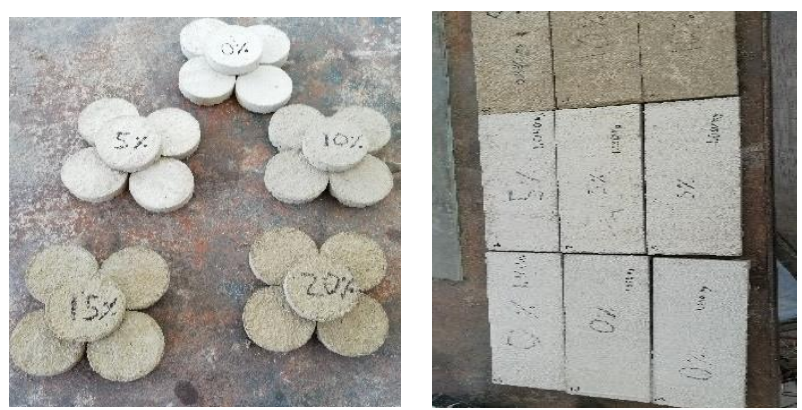

Figura 7 Elaboración de muestras para pruebas térmicas

\section{Resistencia Mecánica}

La resistencia a la compresión del mortero se determinó fracturando las muestras cubicas en una prensa hidráulica para ensayos de compresión, esta se determinó a partir de la carga de ruptura dividida entre la sección que resiste la carga, es decir el área de contacto.

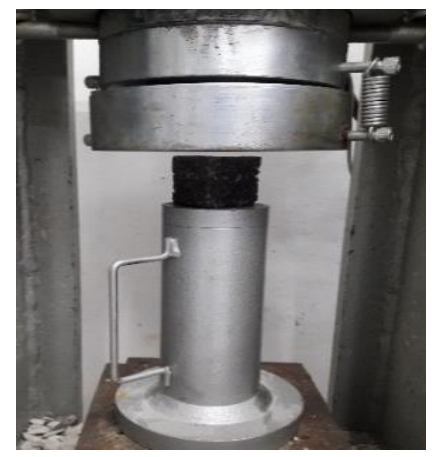

Figura 8 Ensayo de resistencia a la compresión

\section{Conductividad Térmica}

Se evaluó la conducción térmica del mortero por medio del método de medidor de flujo de calor protegido, para el cual se usó el equipo "DTC 300" para mediciones de flujo de calor en materiales. Este equipo emplea un módulo calorimétrico, ubicado en el estante inferior, para la medición y un horno lateral protegido para prevenir pérdidas de calor en los bordes. La resistencia de contacto se minimiza mediante la aplicación de una carga neumática al estante de pruebas y el uso de un compuesto de interfaz con conducción térmica.

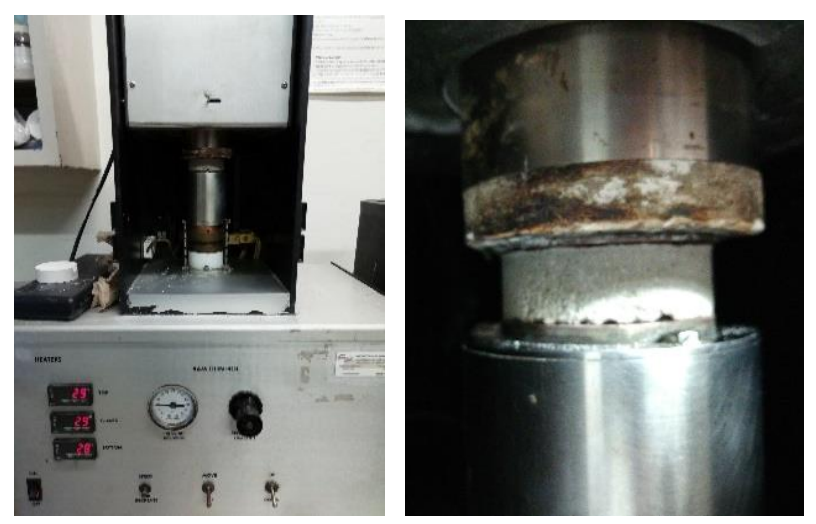

Figura 9 Ensayos de conductividad térmica

\section{Transmitancia Térmica}

La transmitancia térmica es la conductividad del material por el método de convección, el cual se midió por el método de la caja caliente, midiendo el flujo de calor de la placa por media una cámara de transferencia de calor.
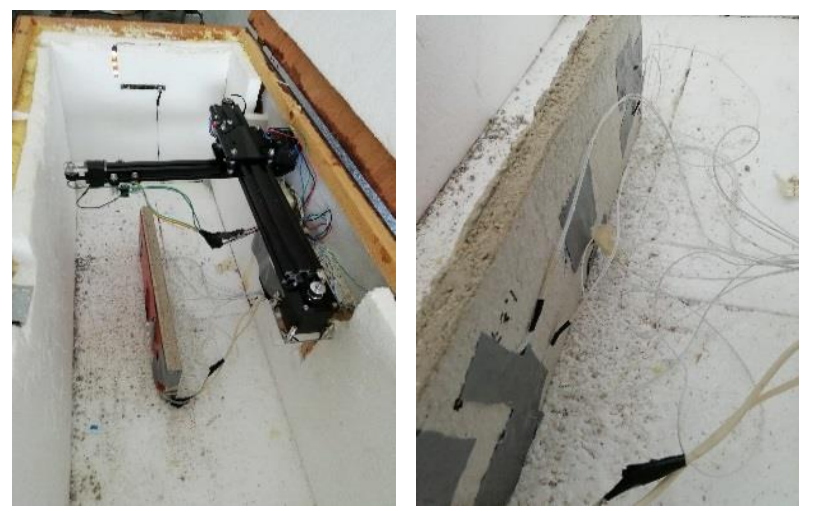

Figura 10 Ensayos de transmitancia térmica

\section{Calor Específico}

La medición del calor específico del mortero se realizó por medio del equipo DSC-Q200 el cual realiza una medición de escaneo de calorimetría diferencial para la obtención de resultados. 
Para este equipo se utilizaron muestras menores a los 10 miligramos, obtenidas de las muestras más grandes al triturarlas.
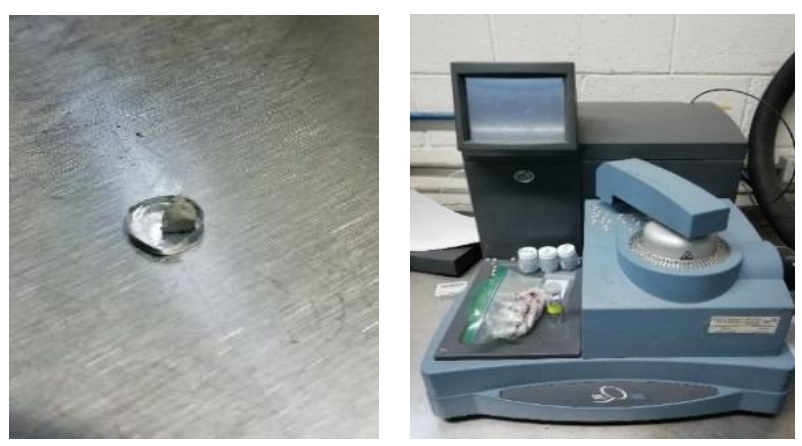

Figura 11 Determinación del Calor Específico

\section{Densidad y Absorción}

La densidad del mortero se determinó por medio del método de la determinación de la densidad aparente midiendo el peso de la muestra y dividiéndola entre el empuje hidrostático que esta misma tiene hacia la superficie, teniendo en cuenta la densidad del líquido auxiliar (fig. 12A). La absorción fue determinada por el método de diferencia de pesos, teniendo el peso de la muestra completamente seca y el peso de la misma sumergido en agua por 24 horas, para determinar su porcentaje de agua absorbida (fig. 12-B).
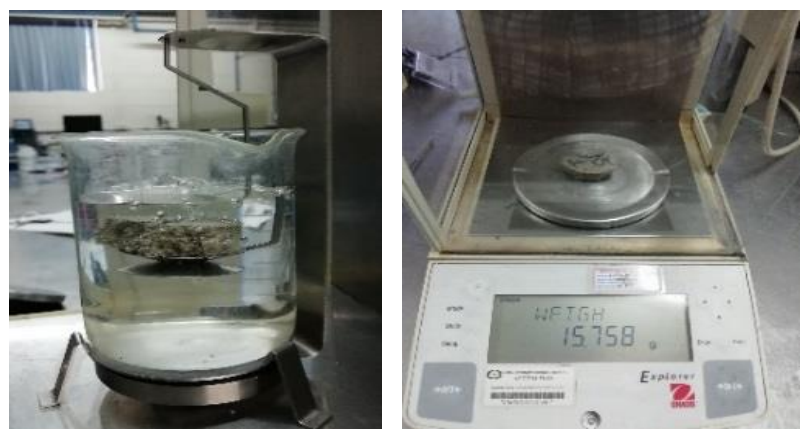

Figura 12 Determinación de la densidad y absorción del mortero

\section{Resultados y Discusión}

\section{Tratamiento del material de sustitución}

Al efectuar el tratamiento del alga se observó una reducción del volumen del material muy significativa y se tomaron las medidas necesarias para obtener un coeficiente de reducción, comparando el volumen del alga en su estado de recolección y el volumen final después de su tratamiento, considerando las pérdidas de material durante su tratamiento, la deshidratación y el cambio en la estructura química después del lavado con $\mathrm{NaOH}$.
Al término del tratamiento del material, obtuvimos un $11.11 \%$ del volumen de la cantidad original del sargazo recolectado, por lo tanto se propone realizar el tratamiento "in situ", es decir tratar el material directamente en el lugar de recolección para economizar gastos en transporte desde el lugar de recolección, logrando de esta manera transportar hasta 10 veces más material útil para su uso como material en el ámbito constructivo.

\section{Caracterización de materiales.}

\section{Caracterización del material de sargazo.}

La caracterización del material después de su tratamiento obtuvo los datos mostrados en el grafico 1 y en la tabla 2 .

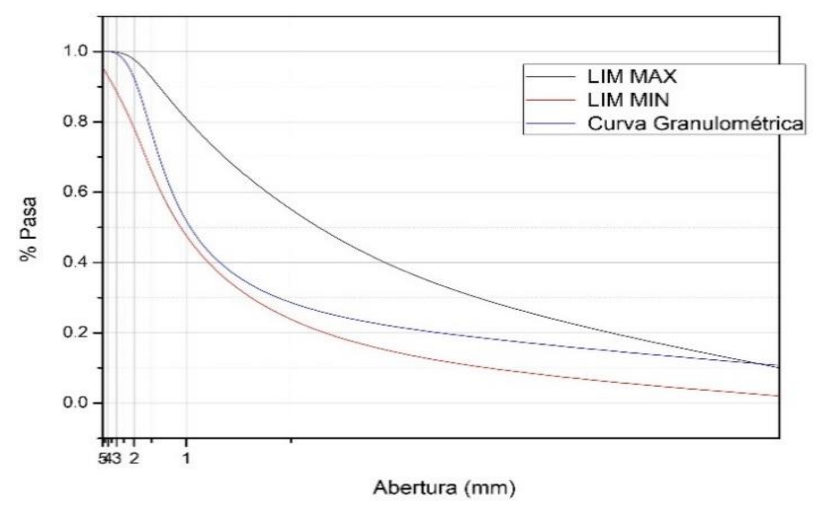

Grafico 1 Curva granulométrica del material de sargazo

\begin{tabular}{|l|l|}
\hline Propiedades & Sargazo tratado \\
\hline Peso específico & $968.25 \mathrm{Kg} / \mathrm{m} 3$ \\
\hline Peso volumétrico seco suelto & $305.06 \mathrm{Kg} / \mathrm{m} 3$ \\
\hline Peso volumétrico seco compacto & $415.54 \mathrm{Kg} / \mathrm{m} 3$ \\
\hline Contenido de humedad & $9.225 \%$ \\
\hline Absorción & $17.72 \%$ \\
\hline Módulo de finura & 2.90 \\
\hline
\end{tabular}

Tabla 2 Resultados de caracterización del material de sargazo

\section{Caracterización del material pétreo}

La caracterización del material pétreo de la región obtuvo los datos mostrados en el grafico 2 y en la tabla 3. Podemos observar que el material resulta ser muy ligero en comparación de otros materiales de construcción, sin embargo el porcentaje de absorción es muy alto, por lo cual existen impedimentos en el colado del material, demostrando una cualidad hidrofílica. 


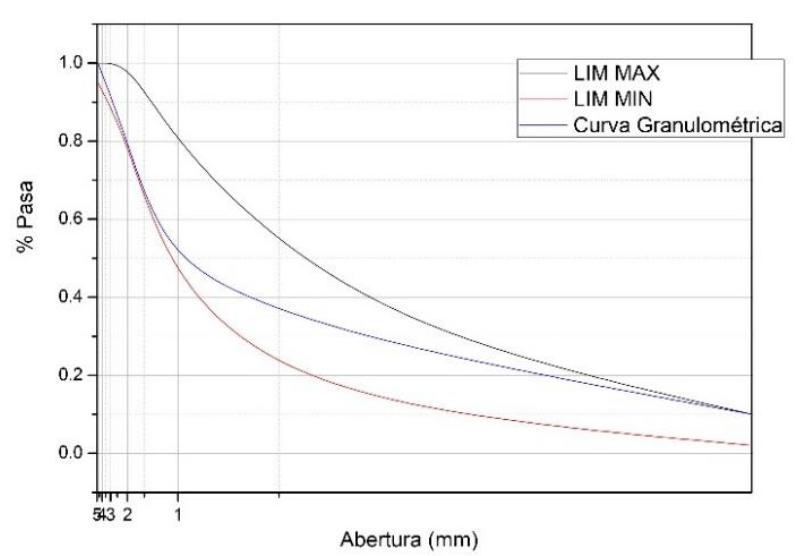

Grafico 2 Curva granulométrica del agregado fino

\begin{tabular}{|l|l|}
\hline Propiedades & Material Pétreo \\
\hline Peso específico & $2898.80 \mathrm{Kg} / \mathrm{m} 3$ \\
\hline Peso volumétrico seco suelto & $1487.50 \mathrm{Kg} / \mathrm{m} 3$ \\
\hline Peso volumétrico seco compacto & $1649.30 \mathrm{Kg} / \mathrm{m} 3$ \\
\hline Contenido de humedad & $0.335 \%$ \\
\hline Absorción & $2.95 \%$ \\
\hline Módulo de finura & 2.93 \\
\hline
\end{tabular}

Tabla 3 Resultados de caracterización del agregado fino

\section{Fluidez}

Se realizó la prueba de fluidez para determinar el contenido mínimo del agua que requiere para que la mezcla sea lo suficientemente fluida y poder medir la plasticidad y trabajabilidad del mortero. Esta prueba consiste en obtener el porcentaje que aumenta el diámetro original de la base de un cono truncado formado previamente con un molde al centro de una mesa de fluidez (fig. 13- A), la medición del diámetro final se realiza después de que la mesa ha sido sometida a una serie de caídas por medios mecánicos (fig. 13-B). El mortero fue diseñado para tener una fluidez de entre 100 y $120 \%$.
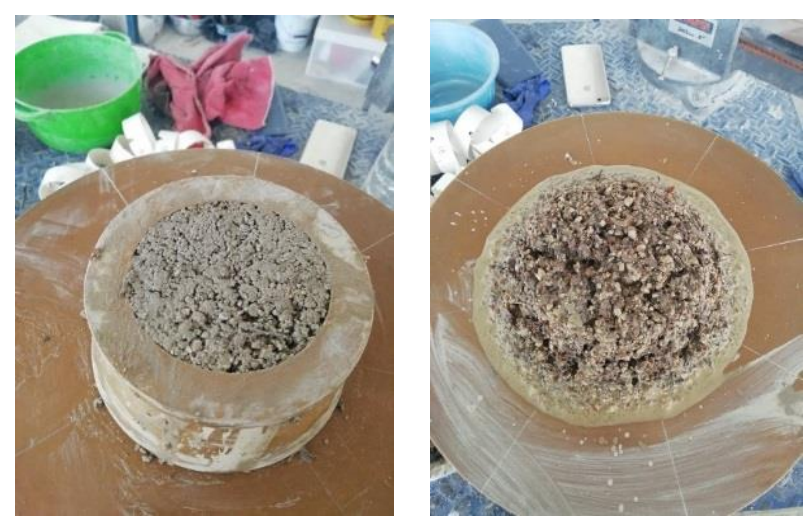

Figura 13 Pruebas de fluidez en el motero
Las muestras con adición del material de sustitución requirieron un aumento en el agua añadida debido a la absorción del material en cuestión.

A mayor porcentaje de sustitución mayor necesidad de agua añadida. Debido a la cantidad de agua que estaba siendo añadida se buscó tener el límite inferior de la fluidez de diseño (entre $100 \%$ y $120 \%$ ) para utilizar la menor cantidad de agua, debido a que la resistencia del mortero es determinada por la relación agua/cemento y a mayor cantidad de agua menor sería la resistencia.

\begin{tabular}{|c|c|c|c|c|}
\hline \multirow{2}{*}{$\begin{array}{l}\text { Porcentaje de } \\
\text { sustitución }\end{array}$} & \multicolumn{2}{|c|}{ Diámetro } & \multirow[b]{2}{*}{ Fluidez } & \multirow{2}{*}{$\begin{array}{l}\text { Agua } \\
\text { añadida }\end{array}$} \\
\hline & Inicial & Final & & \\
\hline $0 \%$ & 7 & 14.5 & $107.14 \%$ & $0.00 \%$ \\
\hline $5 \%$ & 7 & 14.2 & $102.86 \%$ & $39.06 \%$ \\
\hline $10 \%$ & 7 & 14 & $100.00 \%$ & $70.31 \%$ \\
\hline $15 \%$ & 7 & 14 & $100.00 \%$ & $93.75 \%$ \\
\hline $20 \%$ & 7 & 14 & $100.00 \%$ & $117.19 \%$ \\
\hline
\end{tabular}

Tabla 4 Fluidez y porcentajes de agua añadida

\section{Propiedades físicas del mortero endurecido}

Los resultados del mortero de resistencia mecánica, absorción y densidad se muestran en la tabla 5.

\begin{tabular}{|l|l|r|r|r|r|r|r|}
\hline Propiedades & Unidad & \multicolumn{2}{|c|}{$\mathbf{0 \%}$} & $\mathbf{5 \%}$ & $\mathbf{1 0} \%$ & $\mathbf{1 5 \%}$ & $\mathbf{2 0 \%}$ \\
\hline $\begin{array}{l}\text { Resistencia } \\
\text { Mecánica }\end{array}$ & $\mathrm{Kg} / \mathrm{cm}^{2}$ & 192.40 & 75.86 & 23.33 & 9.20 & 7.60 \\
\hline Absorción & $\%$ & 9.18 & 12.77 & 16.25 & 19.56 & 22.22 \\
\hline Densidad & $\mathrm{g} / \mathrm{cm}^{3}$ & 2.027 & 1.868 & 1.753 & 1.711 & 1.684 \\
\hline
\end{tabular}

Tabla 5 Resultados de pruebas físicas del mortero

\section{Resistencia Mecánica}

En el gráfico 3 podemos observar cual es el aumento de cada uno de los porcentajes de sustitución con respecto a los días de curado, en este podemos observar que los porcentajes más altos no tuvieron un aumento significativo en la resistencia, al igual que podemos observar que solo el 5\% alcanzó la resistencia con la cual fue diseñada el mortero $\left(75 \mathrm{~kg} / \mathrm{cm}^{2}\right)$ mientras que el $10 \%$ alcanzó una resistencia ligeramente aceptable para morteros de recubrimiento, mientras que los porcentajes de 15 y $20 \%$ se determinaron como resistencias muy bajas. 


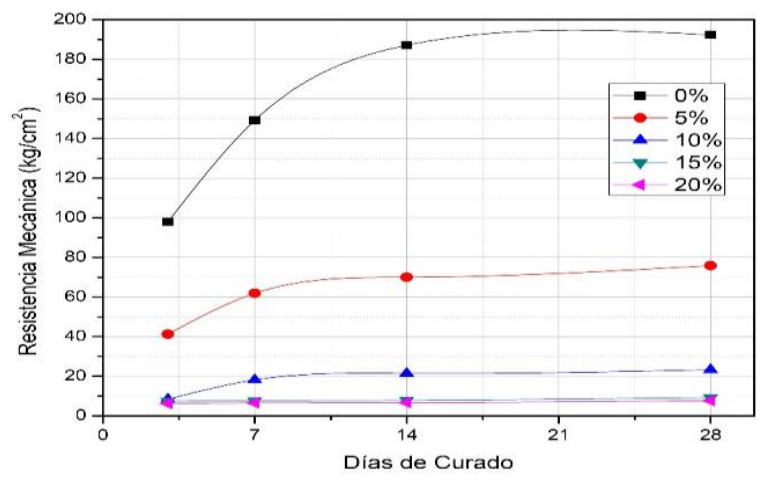

Grafico 3 Aumento de resistencia a la compresión de cada porcentaje de sustitución

En el gráfico 4 podemos observar una caída en la resistencia mecánica del mortero con respecto al aumento del porcentaje de sustitución del agregado, lo cual determina que a mayor uso del alga como sustituto, es menor la resistencia del mortero.

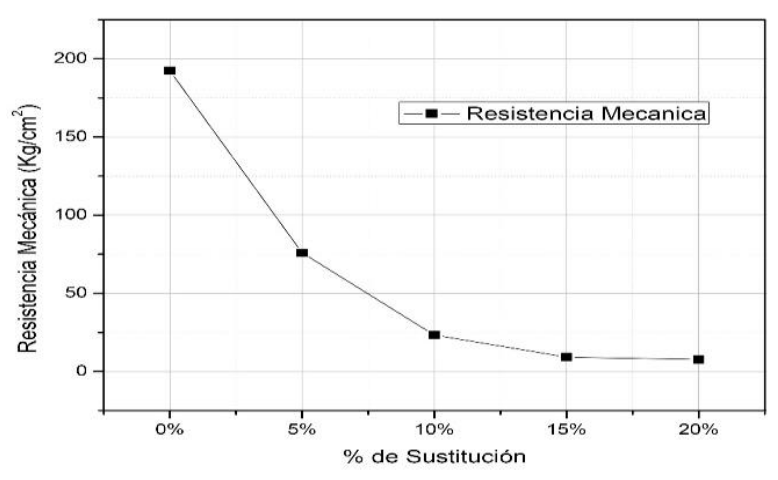

Grafico 4 Resistencia mecánica en relación al aumento de sustitución del agregado pétreo

\section{Densidad}

La densidad es una propiedad estrechamente ligada con la conductividad térmica, Asdrubali (2015) demuestra que a menor densidad, es menor la conductividad térmica del material. En el gráfico 5 podemos observar que a mayor porcentaje de sustitución, es menor la densidad, por lo cual podemos concluir que el mortero es más ligero al tener mayor sustitución y por consiguiente tiene una conductividad térmica menor.

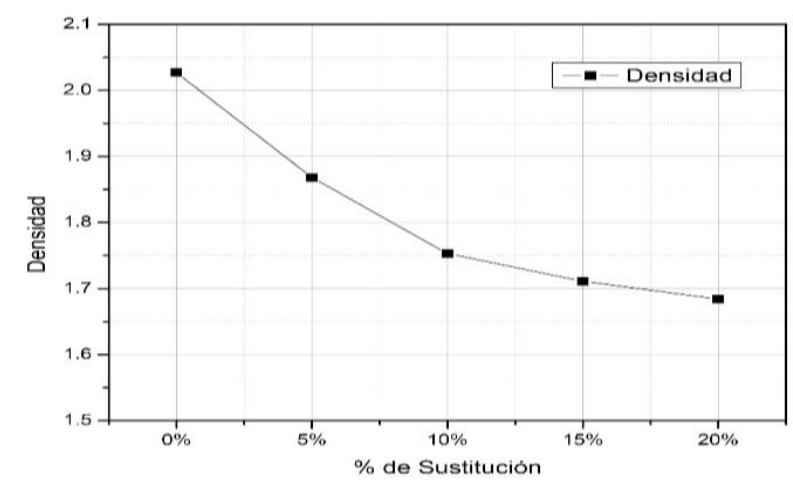

Grafico 5 Densidad de cada porcentaje de sustitución del agregado

\section{Absorción}

La absorción del material se expresa en el gráfico 6 , en el cual se puede observar que a mayor porcentaje de sustitución es mayor la absorción del material, debido a las características hidrofilicas de las fibras del sargazo, lo cual no es adecuado para el muro de recubrimiento, ya que retiene la humedad y puede llevar a la proliferación de hongos.

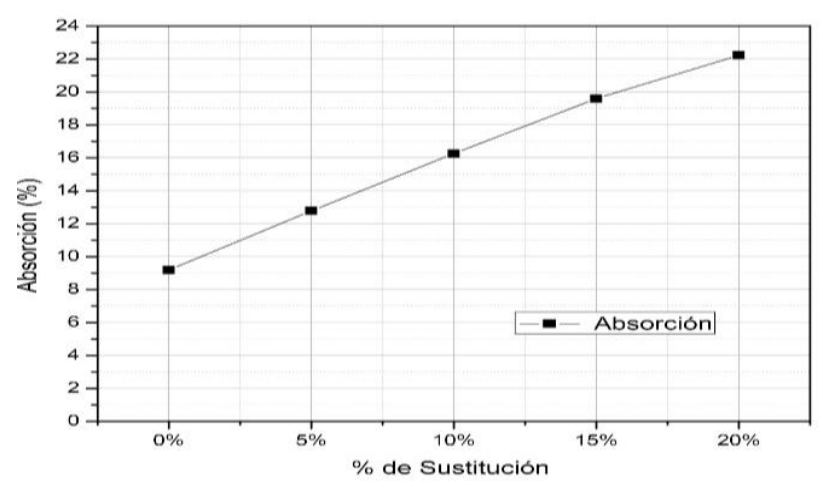

Grafico 6 Absorción de cada porcentaje de sustitución del agregado

\section{Propiedades Térmicas}

A continuación, se muestran los resultados de las propiedades térmicas de conductividad térmica, transmitancia térmica y calor específico, tabla 6.

\begin{tabular}{|l|l|c|c|c|c|c|}
\hline Propiedades & \multicolumn{1}{|c}{ Unidad } & $\mathbf{0 \%}$ & $\mathbf{5 \%}$ & $\mathbf{1 0 \%}$ & $\mathbf{1 5 \%}$ & $\mathbf{2 0 \%}$ \\
\hline $\begin{array}{l}\text { Conductivida } \\
\text { d Térmica } \\
\text { (Conducción) }\end{array}$ & $\mathrm{w} / \mathrm{mK}$ & 0.788 & 0.519 & 0.397 & 0.236 & 0.211 \\
\hline $\begin{array}{l}\text { Transmitanci } \\
\text { a Térmica } \\
\text { (Convección) }\end{array}$ & $\mathrm{w} / \mathrm{mK}$ & 0.630 & 0.536 & 0.523 & 0.474 & 0.405 \\
\hline $\begin{array}{l}\text { Calor } \\
\text { Específico }\end{array}$ & $\mathrm{J} / \mathrm{g}^{\circ} \mathrm{C}$ & 0.884 & 0.893 & 0.902 & 0.911 & 0.920 \\
\hline
\end{tabular}

Tabla 6 Resultados de propiedades térmicas

\section{Conductividad Térmica}

Como se observa en el grafico 7, la conductividad térmica incrementa al aumentar el porcentaje de sustitución, por lo cual se concluye que el uso del alga reduce la transferencia de calor del material por conducción. 


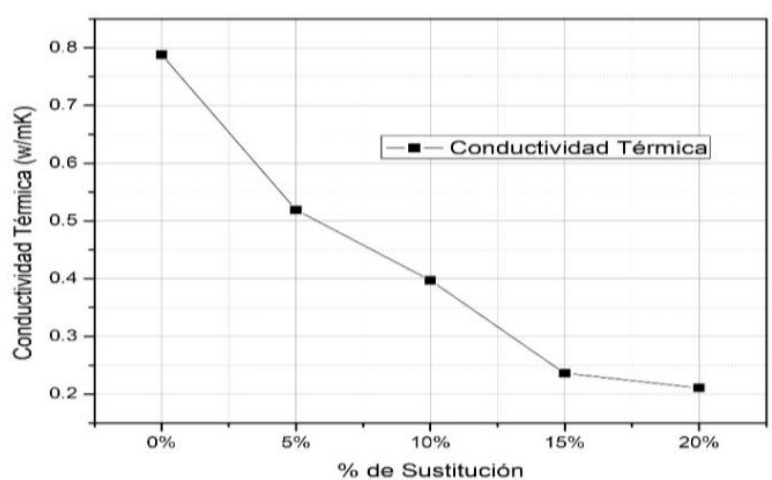

Grafico 7 Conductividad térmica de cada porcentaje de sustitución del agregado

\section{Transmitancia Térmica}

Como se observa en el grafico 8, la conductividad térmica incrementa al aumentar el porcentaje de sustitución, por lo cual se concluye que el uso del alga reduce la transferencia de calor del material por convección.

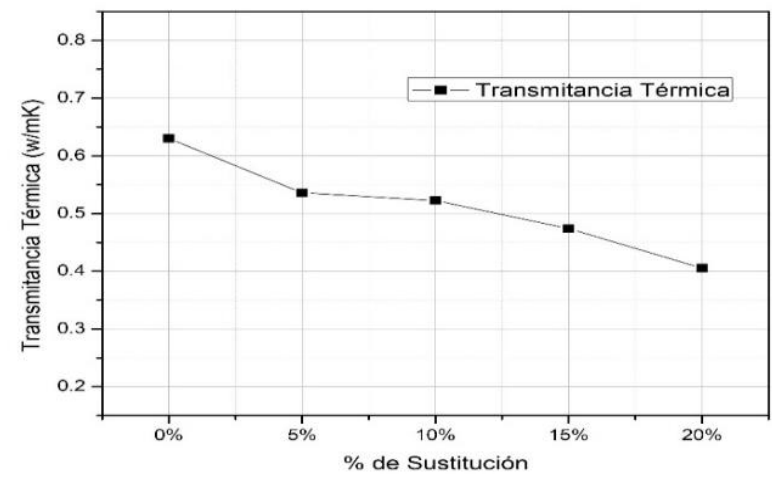

Grafico 8 Transmitancia térmica de cada porcentaje de sustitución del agregado

\section{Calor Específico}

El calor específico aumenta en medida que aumenta la sustitución del agregado en el mortero, lo cual quiere decir que a mayor sargazo, es mayor la energía que el material puede absorber antes de empezar a calentarse, tal y como podemos observar en el grafico 9 .

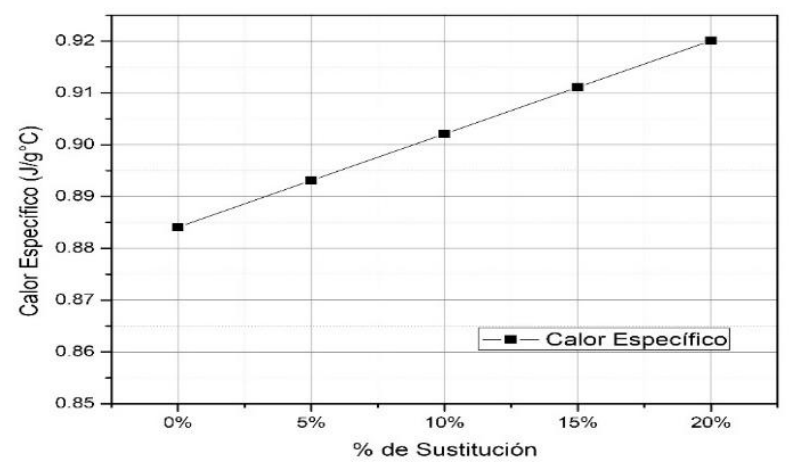

Grafico 9 Calor específico de cada porcentaje de sustitución del agregado

\section{Conclusiones}

Al término del tratamiento, el material corresponde solamente a un $11 \%$ del volumen del material recolectado, por lo tanto es recomendable realizar un tratamiento "In situ" para reducir los costos de transporte del material. Los resultados de la caracterización muestran una similitud en la granulometría del material sustituto con el agregado fino y una gran diferencia en pesos por lo cual se pueden hacer morteros más ligeros para reducir la carga a las estructuras, sin embargo su retención de agua es mayor y afecta la resistencia en los porcentajes de sustitución más elevados.

Debido a las características hidrofílicas del material tratado proveniente del alga de sargazo, el mortero exige una mayor cantidad de agua en su diseño para alcanzar la fluidez requerida, lo cual aumenta la relación agua/cementante del mortero y por consiguiente reduce su resistencia.

Los resultados en las características térmicas fueron satisfactorios, a mayor material de sargazo como sustitución del agregado pétreo, menor es la transferencia de calor tanto por conducción como por convección, lo cual significa que la temperatura interior de una edificación puede durar más tiempo antes de calentarse por las incidencias solares. De igual manera los resultados del calor especifico fueron satisfactorios, al aumentar el calor específico logramos que el material requiera una mayor energía para calentarse, lo cual es una solución para la retención de calor en los muros de concreto.

Analizando los resultados térmicos y los mecánicos del mortero podemos concluir que a pesar de tener una reducción en la resistencia a la compresión, es posible utilizar el sargazo como sustituto del agregado fino, en porcentajes muy bajos, teniendo como porcentaje ideal el de $5 \%$, en el cual la resistencia es aceptable y el mejoramiento térmico es notable.

Para su uso como mortero de recubrimiento con aislamiento térmico es necesario reducir el rango de porcentajes de sustitución, en los cuales la resistencia y adherencia sean óptimas para su aplicación, proponiendo un nuevo rango de investigación entre 0 y $10 \%$. 
El principal problema del sargazo para ser eficiente en construcción es su absorción, por el cual el agua se almacena en el muro creando zonas de proliferación de hongos o bacterias y al liberar esta misma quedan espacios vacíos los cuales provocan una caída en la resistencia.

\section{Agradecimientos}

$\mathrm{Al}$ TecNM por el apoyo que se le proporciona a los investigadores y el financiamiento de los proyectos.

Al Consejo Nacional de Ciencia y Tecnología por el apoyo financiero que brinda su sistema de becas del programa de nacional de posgrados de calidad para la investigación y el desarrollo tecnológico.

Al Centro de Investigación de Materiales Avanzados y al Dr. Daniel Lardizábal Gutiérrez por el apoyo técnico en las mediciones de las propiedades térmicas del material y el asesoramiento en los temas relacionados con las mismas.

\section{Referencias bibliográficas}

[1] F. Asdrubali, F. D'Alessandro, S. Schiavoni. A review of unconventional sustainable building insulation materials.4 (2015) 1-17.

[2] V. Corinaldesi, A. Mazzoli, R. Siddique. Characterization of lightweight mortars containing Wood processing by-products waste. 123 (2016) 281-289.

[3] F. Fernández, C. J. Boluda, J. Olivera, L. A. Guillermo, B. Gómez, E. Echavarría, A. M. Gómez. Análisis elemental prospectivo de la biomasa algal acumulada en las costas de la republica dominicana durante 2015. 44 (2017) 11-22.

[4] N. U. Kockal. Investigation about the effect of different fine aggregates on physical, mechanical and thermal properties of mortars. 124 (2016) $816-825$.

[5] N. Benmansour, B. Agoudjil, A. Gherabli, A. Kareche, A. Boundenne. Thermal and mechanical performance of natural mortar reinforced with date palm fibers for use as insulating materials in building. 81 (2014) 98104.
[6] S. O. G. Osseni, C. Ahouannou, E. A. Sanya, Y. Jannot. Investigation on the use of the cemente mortar containing banana fibers as thermal insulator in building. 4 (2016) 11421152

[7] L. F. Liu, H. Q. Li, A. Lazzaretto, G. Manente, C. Y. Tong, Q. B. Liu, N. P. Li. The development history and prospects of biomassbased insulation materials for buildings. 69 (2017) 912-932.

[8] I. Boulaoued, I. Amara, A. Mhimid. Experimental Determination of Thermal Conductivity and Diffusivity of New Building Insulating Materials. 34 (2016) 325-331.

[9] B. I. Van Tussenbroek, H. A. Hernandez, R. E. Rodriguez, J. Espinoza, H. M. Canizales, C. E. Gonzáles. M. G. Barba-Santos, A. Vega, L. Collado. Severe impacts of Brown tides caused by Sargassum spp. On near Caribbean seagrass communities. 122 (2017) 272-281.

[10] M. G. Gomes, I. Flores, L. M. Manga, A. Soares, J. de Brito. The influence of moisture content on the termal conductivity of external thermal mortars. 135 (2017) 279-286.

[11]S. Widolo, F. Ma'arif, B. S. Gan. Thermal conductivity and compressive strength of lightweight mortar utilizing Pumice breccia as fine aggregate. 171 (2017) 768-773.

[12] R. E. Rodriguez- Martinez, B. V. Tussenbroek, E. Jordán- Dahlgren. Afluencia masiva de sargazo pelágico a la costa del caribe mexicano. (2017)

[13] L. A. G. Puc-Ortiz (2009). Caracterización térmica de morteros utilizados en acabados de edificaciones y viviendas en la ciudad de Chetumal Quintana Roo. Tesis de Maestría. Instituto Tecnológico de Chetumal. Quintana Roo, México. p. 4.

[14] S. O. G. Osseni, C. Ahouannou, E. A. Sanya, Y. Jannot. Hot plate method with two simultaneous temperature measurements for themal characterization of building material. (2017) 38-81.

[15] [4] M. Chikhi, B. Agoudjil, A. Boudenne, A. Gherabli. Experimental investigation of a new biocomposite with low cost for thermal insulation. 66(2013) 267-273 